\title{
Cluster Expansion for the Dielectric Constant of a Polarizable Suspension
}

\author{
B. U. Felderhof, ${ }^{1}$ G. W. Ford, ${ }^{2}$ and E. G. D. Cohen $^{3}$
}

Received August 24, 1981

\begin{abstract}
We derive a cluster expansion for the electric susceptibility kernel of a dielectric suspension of spherically symmetric inclusions in a uniform background. This also leads to a cluster expansion for the effective dielectric constant. It is shown that the cluster integrals of any order are absolutely convergent, so that the dielectric constant is well defined and independent of the shape of the sample in the limit of a large system. We compare with virial expansions derived earlier in statistical mechanics for the dielectric constant of a nonpolar gas. In these expansions the virial coefficients are given by integrals which are only conditionally convergent.
\end{abstract}

KEY WORDS: Cluster expansion; virial coefficients; dielectric constant; polarizability; suspension; shape dependence; absolute convergence; Clausius-Mossotti formula.

\section{INTRODUCTION}

This will be the first in a series of papers on the problem of finding the effective dielectric constant of a system of nonoverlapping spherically symmetric polarizable inclusions statistically distributed in an otherwise homogeneous medium. The problem can equivalently be formulated for the effective magnetic permeability of a paramagnetic material, or for the effective heat conductivity or electrical conductivity of a medium with similar inclusions. We use the dielectric formulation because it allows a clear intuitive picture. The problem is a relatively simple one in a range of problems concerned with the effective transport properties of heterogeneous continuous media, for example the effective viscosity of a fluid

\footnotetext{
${ }^{1}$ Institut für Theoretische Physik A, RWTH Aachen, 5100 Aachen, West Germany.

${ }^{2}$ Department of Physics, University of Michigan, Ann Arbor, Michigan, USA.

${ }^{3}$ Rockefeller University, New York, New York, USA.
} 
suspension or the effective elastic constants of a solid suspension. Some excellent reviews of the subject have appeared recently. ${ }^{(1-4)}$ For earlier work on dielectrics we refer to the review by Brown. ${ }^{(5)}$

The dielectric problem is equivalent on the microscopic level to that of finding the dielectric constant of a system of nonpolar molecules in thermal equilibrium, the molecules playing the role of the inclusions. This problem has been extensively studied in equilibrium statistical mechanics, usually neglecting all but dipolar interactions between the molecules. In early work by Kirkwood ${ }^{(6)}$ and Yvon ${ }^{(7)}$ the Clausius-Mossotti function $\left(\epsilon^{*}-1\right)$ $/\left(\epsilon^{*}+2\right)$, where $\epsilon^{*}$ is the effective dielectric constant, was expanded in powers of the polarizability of the particles. This must be regarded as a weak-coupling expansion. In later theories ${ }^{(8,9)}$ this function was expanded in powers of the density fluctuations, but these are also weak-coupling expansions. Buckingham and Pople ${ }^{(10)}$ were the first to expand the Clausius-Mossotti function in powers of density. This expansion is called the dielectric virial expansion in analogy to the virial expansion for the pressure. It is a strong-coupling expansion, since the dielectric response of individual molecules is not treated in perturbation theory. It was shown by Hill, ${ }^{(1)}$ Kaufman and Watson, ${ }^{(12)}$ and by Isihara ${ }^{(13,14)}$ how such a virial expansion can be obtained from the usual cluster expansions for the thermodynamic properties of a fluid in the presence of an external field. We shall show in this article, however, that such virial expansions, while in principle correct, are inherently unsatisfactory, since the cluster integrals which appear are not absolutely convergent. The existence of $\epsilon^{*}$ in equilibrium statistical mechanics and in dipole approximation was shown by Wertheim ${ }^{(15)}$ by means of a graphical analysis, following earlier work by Ramshaw. (16)

The difficulty with these equilibrium statistical mechanical methods is that they always involve formation of the partition function for a system in a given external applied field $\mathbf{E}_{0}(\mathbf{r})$. By appropriate (functional) differentiation one then gets an expression for $\langle\mathbf{P}(\mathbf{r})\rangle$, the average polarization in the medium, in terms of $\mathbf{E}_{0}(\mathbf{r})$. But, as is well known from macroscopic electrostatics, this expression is necessarily very nonlocal and in particular depends upon the shape of the sample. It is this shape dependence which leads to the conditional convergence of the various cluster integrals. It is clear, however, that what is wanted is the relation between $\langle\mathbf{P}(\mathbf{r})\rangle$ and $\langle\mathbf{E}(\mathbf{r})\rangle$, the average electric field within the sample. This relation should be local in character and shape independent, but is not easy to get out of the theory.

The method we use to get the relation between $\langle\mathbf{P}\rangle$ and $\langle\mathbf{E}\rangle$ is based on that outlined in a study by one of us (G.W.F.) on a time-dependent formulation of multiple scattering in disordered systems. ${ }^{(17)}$ That method 
was based in turn on a related method introduced by another of us (E.G.D.C.) in connection with the derivation of the Boltzmann equation for moderately dense gases. ${ }^{(18)}$ The strategy of the method as applied to the dielectric problem is as follows. We express the electric field in the presence of a fixed configuration of inclusions as a formal operator acting on a general applied field $\mathbf{E}_{0}(\mathbf{r})$ and then average over the configurations of the inclusions to get an expression for $\langle\mathbf{E}\rangle$. In the same way we obtain an expression for $\langle\mathbf{P}\rangle$, again expressed as the configuration average of a formal operator acting on $\mathbf{E}_{0}$. We then use cluster expansions to eliminate $\mathbf{E}_{0}$ to obtain the desired relation between $\langle\mathbf{P}\rangle$ and $\langle\mathbf{E}\rangle$.

We have been anticipated in this treatment of the problem by Finkel'berg, ${ }^{(19)}$ who in a short paper sketched what is essentially the above method but gave very few details. In a later paper he gave an explicit expression for the second order term in $\epsilon^{*}{ }^{(20)}$ but in a slightly different form from ours since he assumed a uniform applied field. The same expression for the second-order term was found by Jeffrey, ${ }^{(21)}$ who used a method due to Batchelor to make the integral absolutely convergent. ${ }^{(22)}$ The equivalence of our form for the second-order term and theirs will be shown in a following article devoted to the explicit calculation of that term. Jeffrey has also extended Batchelor's method to higher order, ${ }^{(23)}$ but his discussion is marred by the fact that an essential relation he uses, the identification of the average field $\langle\mathbf{E}\rangle$ and the applied field $\mathbf{E}_{0}$, is clearly incorrect. Nevertheless, the expansion he obtains is very close to ours, as we shall show in detail in a later article.

In Section 2 we formulate the basic equations for the dielectric problem. In Section 3 we write down cluster expansions for the average polarization and the average field. By elimination of the external field we find the cluster expansion for the electrical susceptibility kernel. In Section 4 we introduce the binary collision expansion which we use to discuss the cluster properties of the operators which appear in the cluster integrals. In Section 5 we rearrange the integrands of the cluster integrals, expressing them as a sum of terms, each containing a product of a so-called block distribution function and a chain operator. This rearrangement is used in Section 6 where we prove the absolute convergence of each of these terms. In Section 7 we discuss the cluster expansion of the effective dielectric constant. In Section 8 we compare our results with those obtained from the usual virial expansions of statistical mechanics.

\section{FORMULATION OF THE PROBLEM}

Our aim is to determine the effective dielectric properties of a medium with a locally varying dielectric constant $\epsilon(\mathbf{r})$. Specifically, we assume $\epsilon(\mathbf{r})$ is 
uniform and equal to a constant $\epsilon_{1}$ except within a set of $N$ nonoverlapping spherical inclusions. The inclusions are identical, each of radius $a$ and characterized by a spherically symmetric dielectric constant. For a fixed configuration of inclusions in which they are centered at $\mathbf{R}_{1}, \mathbf{R}_{2}, \ldots, \mathbf{R}_{N}$, the dielectric constant at a field point $\mathbf{r}$ is then

$$
\epsilon(1, \ldots, N ; \mathbf{r})= \begin{cases}\epsilon_{1} & \left|\mathbf{r}-\mathbf{R}_{j}\right|>a, \quad j=1, \ldots, N \\ \epsilon\left(\left|\mathbf{r}-\mathbf{R}_{j}\right|\right), & \left|\mathbf{r}-\mathbf{R}_{j}\right|<a\end{cases}
$$

Here we have introduced a shorthand notation in which we indicate only the labels of the coordinates specifying the configuration. This notation will be used throughout this paper. The basic equations for the electric field $\mathbf{E}$ and the dielectric displacement $\mathbf{D}$ are Maxwell's electrostatic equations:

$$
\operatorname{div} \mathbf{D}=4 \pi \rho_{0}, \quad \operatorname{curl} \mathbf{E}=0, \quad \mathbf{D}=\epsilon \mathbf{E}
$$

where $\rho_{0}=\rho_{0}(\mathbf{r})$ is a fixed charge distribution, independent of the configuration of the inclusions, which is the source of the fields.

The applied electric field $\mathbf{E}_{0}=\mathbf{E}_{0}(\mathbf{r})$ is the solution of the equations (2.2) with $\epsilon$ a uniform dielectric constant $\epsilon_{1}$. The electric field $\mathbf{E}(1, \ldots, N)$ $=\mathbf{E}(1, \ldots, N ; \mathbf{r})$ in the presence of a fixed configuration of $N$ inclusions is the solution of the equations (2.2) with the same charge distribution $\rho_{0}$ but with dielectric constant $\epsilon$ given by (2.1). Because of the linearity of the electrostatic equations the field $\mathbf{E}(1, \ldots, N)$ in the presence of the inclusions and the applied field are linearly related,

$$
\mathbf{E}(1, \ldots, N ; \mathbf{r})=\int d \mathbf{r}^{\prime} \mathrm{K}\left(\mathbf{r}, \mathbf{r}^{\prime} ; 1, \ldots, N\right) \cdot \mathbf{E}_{0}\left(\mathbf{r}^{\prime}\right)
$$

or, in a still shorter notation,

$$
\mathbf{E}(1, \ldots, N)=\mathrm{K}(1, \ldots, N) \cdot \mathbf{E}_{0}
$$

We emphasize that the meaning of this formal operator $\mathrm{K}(1, \ldots, N)$ is no more than that $\mathbf{E}(1, \ldots, N)$ is the solution of the electrostatic equations in the presence of a fixed configuration of inclusions, while $\mathbf{E}_{0}$ is the solution of these equations with the same source in the absence of inclusions.

The dielectric displacement in the presence of a fixed configuration of inclusions is

$$
\mathbf{D}(1, \ldots, N)=\epsilon(1, \ldots, N) \mathbf{E}(1, \ldots, N)
$$

We also introduce the induced polarization, relative to the medium in the absence of inclusions, via the relation

$$
\mathbf{D}(1, \ldots, N)=\epsilon_{1} \mathbf{E}(1, \ldots, N)+4 \pi \mathbf{P}(1, \ldots, N)
$$

Using (2.5) and (2.4) we can then write

$$
\mathbf{P}(1, \ldots, N)=\chi(1, \ldots, N) \mathrm{K}(1, \ldots, N) \cdot \mathbf{E}_{0}
$$


where

$$
\chi(1, \ldots, N)=\frac{\epsilon(1, \ldots, N)-\epsilon_{1}}{4 \pi}
$$

is the relative dielectric susceptibility. Clearly $\chi$ and, therefore, $\mathbf{P}$, vanish except within the inclusions. It should also be intuitively clear that the induced polarization depends only upon the field $\mathbf{E}_{0}(\mathbf{r})$ within the inclusions.

The inclusions are randomly distributed as described by a probability distribution $W(1, \ldots, N)$, such that $W(1, \ldots, N) d \mathbf{R}_{1} \ldots d \mathbf{R}_{N}$ is the probability of finding a configuration in which inclusion 1 is centered in volume $d \mathbf{R}_{1}$ about $\mathbf{R}_{1}, \ldots$, and inclusion $N$ is centered in volume $d \mathbf{R}_{N}$ about $\mathbf{R}_{N}$. The distribution is assumed normalized to unity and symmetric in the labels $1, \ldots, N$. The partial distribution functions

$$
n(1, \ldots, s)=\frac{N !}{(N-s) !} \int \ldots \int d \mathbf{R}_{s+1} \ldots d \mathbf{R}_{N} W(1, \ldots, N)
$$

give the probability of finding a configuration of $s$ inclusions whatever the configuration of the remaining $N-s$ inclusions. Thus $n(1)$ is the density of inclusions, $n(1,2)$ is the distribution of pairs of inclusions, etc. The assumption that the inclusions do not overlap means that

$$
W(1, \ldots, N)=0 \text { if }\left|\mathbf{R}_{j}-\mathbf{R}_{k}\right|<2 a
$$

for any pair of labels $j, k$. This in turn implies the same property for the partial distribution functions

$$
n(1, \ldots, s)=0 \quad \text { if } \quad\left|\mathbf{R}_{j}-\mathbf{R}_{k}\right|<2 a
$$

for any pair of labels $j, k$ such that $1 \leqslant j<k \leqslant s$.

The average electric field in the presence of the inclusions is

$$
\langle\mathbf{E}\rangle=\int \cdots \int d \mathbf{R}_{1} \ldots d \mathbf{R}_{N} W(1, \ldots, N) \mathbf{E}(1, \ldots, N)
$$

This configuration-averaged field will in general still be position dependent, the field $\mathbf{E}(1, \ldots, N)=\mathbf{E}(1, \ldots, N ; \mathbf{r})$ depends upon the position $\mathbf{r}$ and the configuration of inclusions. In the same way we introduce the average dielectric displacement and the average polarization

$$
\begin{aligned}
\langle\mathbf{D}\rangle & =\int \cdots \int d \mathbf{R}_{1} \ldots d \mathbf{R}_{N} W(1, \ldots, N) \mathbf{D}(1, \ldots, N) \\
\langle\mathbf{P}\rangle & =\int \cdots \int d \mathbf{R}_{1} \ldots d \mathbf{R}_{N} W(1, \ldots, N) \mathbf{P}(1, \ldots, N)
\end{aligned}
$$

These average fields will be related via the average of (2.6),

$$
\langle\mathbf{D}\rangle=\epsilon_{1}\langle\mathbf{E}\rangle+4 \pi\langle\mathbf{P}\rangle
$$


The dielectric properties of the average medium in the presence of inclusions are characterized by the relation between $\langle\mathbf{D}\rangle$ and $\langle\mathbf{E}\rangle$ or, equivalently, the relation between $\langle\mathbf{P}\rangle$ and $\langle\mathbf{E}\rangle$. Because of the linearity of the basic equations, this relation will be linear:

$$
\langle\mathbf{P}\rangle=\mathbf{X} \cdot\langle\mathbf{E}\rangle
$$

The electrical susceptibility operator $X$ will in general be nonlocal, so the relation (2.15) can be expressed, exhibiting explicitly the vector indices $(j, k)$ and field dependences $\left(\mathbf{r}, \mathbf{r}^{\prime}\right)$, in the form

$$
\left\langle P_{j}(\mathbf{r})\right\rangle=\sum_{k=1}^{3} \int d \mathbf{r}^{\prime} X_{j k}\left(\mathbf{r}, \mathbf{r}^{\prime}\right)\left\langle E_{k}\left(\mathbf{r}^{\prime}\right)\right\rangle
$$

In the next section we use cluster expansions of the operator $\mathrm{K}(1, \ldots, N)$ to obtain an explicit formal expression for the susceptibility operator $\mathrm{X}$.

\section{CLUSTER EXPANSION}

Inserting the formal expression (2.4) in the definition (2.12) of the average electric field, we obtain

$$
\langle\mathbf{E}\rangle=\int \cdots \int d \mathbf{R}_{1} \ldots d \mathbf{R}_{N} W(1, \ldots, N) \mathrm{K}(1, \ldots, N) \cdot \mathbf{E}_{0}
$$

Similarly, inserting (2.7) in the definition (2.13) of the average polarization, we get

$$
\langle\mathbf{P}\rangle=\int \cdots \int d \mathbf{R}_{1} \ldots d \mathbf{R}_{N} W(1, \ldots, N) \chi(1, \ldots, N) \mathrm{K}(1, \ldots, N) \cdot \mathbf{E}_{0}
$$

But for nonoverlapping inclusions, using the definitions (2.8) and (2.1), the susceptibility can be written

$$
\chi(1, \ldots, N)=\sum_{j=1}^{N} \chi(j)
$$

where $\chi(j)$ is the susceptibility in the presence of a single inclusion centered at $\mathbf{R}_{j}$. Putting this in (3.2) and using the symmetry of $W(1, \ldots, N)$ and $\mathrm{K}(1, \ldots, N)$ under interchange of the labels, we see that each term in the sum gives the same contribution, so we obtain

$$
\langle\mathbf{P}\rangle=N \int \cdots \int d \mathbf{R}_{1} \ldots d \mathbf{R}_{N} W(1, \ldots, N) \chi(1) \mathrm{K}(1, \ldots, N) \cdot \mathbf{E}_{0}
$$

Our goal is to eliminate the applied field $\mathbf{E}_{0}$ between this expression and the expression (3.1) for $\langle\mathbf{E}\rangle$ to obtain a linear relation of the form $(2.15)$ between the averages $\langle\mathbf{P}\rangle$ and $\langle\mathbf{E}\rangle$. We do this by first introducing cluster expansions of $\mathrm{K}(1, \ldots, N)$ and $\chi(1) \mathrm{K}(1, \ldots, N)$ in the relations (3.1) and 
(3.4) expressing $\langle\mathbf{E}\rangle$ and $\langle\mathbf{P}\rangle$ in terms of $\mathbf{E}_{0}$. These relations then become expansions with their terms ordered according to the number of inclusions involved. We then eliminate $\mathbf{E}_{0}$ between the two series to obtain the relation between $\langle\mathbf{P}\rangle$ and $\langle\mathbf{E}\rangle$ again in the form of a series ordered according to the number of inclusions.

In the case of the expression (3.1) for $\langle\mathbf{E}\rangle$ we introduce cluster operators $L$ defined successively as follows:

$$
\begin{aligned}
K(\varnothing) & =L(\varnothing)=1 \\
K(1) & =L(1)+L(\varnothing) \\
K(1,2)=L(1,2)+L(1)+L(2)+L(\varnothing) & \ldots, \text { etc. }
\end{aligned}
$$

Here $\emptyset$ denotes the empty set, so $K(\varnothing)$ is the identity 1 . The general rule is

$$
K(\Re)=\sum_{\pi \in \Re} L(\Re)
$$

where $\mathfrak{R}$ is a set of inclusion labels and the sum is over all subsets of $\mathscr{\varkappa}$. The inverse of this rule is

$$
\mathrm{L}(\mathscr{\Re})=\sum_{\mathscr{R} \in \mathscr{R}}(-1)^{N-M} \mathrm{~K}(\mathscr{N})
$$

where $N$ and $M$ are, respectively, the number of labels in $\pi$ and $\Re$. Inserting (3.6) in (3.1), remembering that the number of subsets of $s$ objects out of $N$ objects is $[N ! /(N-s) ! s !]$, and using the definition (2.9) of the partial distribution functions, we obtain

$$
\langle\mathbf{E}\rangle=\sum_{s=0}^{N} \frac{1}{s !} \int \cdots \int d \mathbf{R}_{1} \ldots d \mathbf{R}_{s} n(1, \ldots, s) \mathrm{L}(1, \ldots, s) \cdot \mathbf{E}_{0}
$$

In the case of the expression (3.4) for $\langle\mathbf{P}\rangle$ the label 1 is singled out, so we introduce what we term rooted cluster operators $M$ defined successively as follows:

$$
\begin{aligned}
& \chi(1) \mathrm{K}(1)=\mathrm{M}(1) \\
& \chi(1) \mathrm{K}(1,2)=\mathrm{M}(1 ; 2)+\mathrm{M}(1) \\
& \chi(1) \mathrm{K}(1,2,3)=\mathrm{M}(1 ; 2,3)+\mathrm{M}(1 ; 2)+\mathrm{M}(1 ; 3)+\mathrm{M}(1) \\
& \ldots, \text { etc. }
\end{aligned}
$$

The general rule is

$$
\chi(1) \mathrm{K}(1, \Re)=\sum_{\mathscr{N} \subset \mathscr{T}} \mathrm{M}(1 ; \Re)
$$

where $\mathscr{T}$ is a set of labels not including the label 1 and the sum is over all 
subsets of $\Re$. The inverse of this rule is

$$
\mathrm{M}(1 ; \Re)=\chi(1) \sum_{\Re \subset \Re}(-1)^{N-M} \mathrm{~K}(1, \mathfrak{\Re})
$$

Putting the expansion (3.10) in (3.4), we obtain

$$
\langle\mathbf{P}\rangle=\sum_{s=1}^{N} \frac{1}{(s-1) !} \int \cdots \int d \mathbf{R}_{1} \ldots d \mathbf{R}_{s} n(1, \ldots, s) \mathrm{M}(1 ; 2, \ldots, s) \cdot \mathbf{E}_{0}
$$

which is the expansion of the average polarization.

We next eliminate $\mathbf{E}_{0}$ between the series (3.8) and (3.12). The structure of the result is perhaps seen most clearly if we do this explicitly for the first few terms in the series. We will then state the general result. The first few terms in (3.8) are

$$
\langle\mathbf{E}\rangle=\mathbf{E}_{0}+\int d \mathbf{R}_{1} n(1) \mathrm{L}(1) \cdot \mathbf{E}_{0}+\frac{1}{2} \iint d \mathbf{R}_{1} d \mathbf{R}_{2} n(1,2) \mathrm{L}(1,2) \cdot \mathbf{E}_{0}+\cdots
$$

Solving by iteration for $\mathbf{E}_{0}$, we find

$$
\begin{aligned}
\mathbf{E}_{0}= & \langle\mathbf{E}\rangle-\int d \mathbf{R}_{1} n(1) \mathrm{L}(1)\langle\mathbf{E}\rangle \\
& -\frac{1}{2} \iint d \mathbf{R}_{1} d \mathbf{R}_{2}[n(1,2) \mathrm{L}(1,2)-2 n(1) n(2) \mathrm{L}(1) \cdot \mathrm{L}(2)] \cdot\langle\mathbf{E}\rangle+\cdots
\end{aligned}
$$

The first few terms in (3.12) are

$$
\begin{aligned}
\langle\mathbf{P}\rangle= & \int d \mathbf{R}_{1} n(1) \mathrm{M}(1) \cdot \mathbf{E}_{0}+\iint d \mathbf{R}_{1} d \mathbf{R}_{2} n(1,2) \mathrm{M}(1 ; 2) \cdot \mathbf{E}_{0} \\
& +\frac{1}{2} \iiint d \mathbf{R}_{1} d \mathbf{R}_{2} d \mathbf{R}_{3} n(1,2,3) \mathrm{M}(1 ; 2,3) \cdot \mathbf{E}_{0}+\cdots
\end{aligned}
$$

Inserting (3.14) into (3.15), we get the first few terms in the relation between $\langle\mathbf{P}\rangle$ and $\langle\mathbf{E}\rangle$,

$$
\begin{aligned}
\langle\mathbf{P}\rangle= & \int d \mathbf{R}_{1} n(1) \mathrm{M}(1) \cdot\langle\mathbf{E}\rangle \\
& +\iint d \mathbf{R}_{1} d \mathbf{R}_{2}[n(1,2) \mathrm{M}(1 ; 2)-n(1) n(2) \mathrm{M}(1) \cdot \mathrm{L}(2)] \cdot\langle\mathbf{E}\rangle \\
& +\frac{1}{2} \iiint d \mathbf{R}_{1} d \mathbf{R}_{2} d \mathbf{R}_{3} \\
& \times[n(1,2,3) \mathrm{M}(1 ; 2,3)-2 n(1,2) n(3) \mathrm{M}(1 ; 2) \cdot \mathrm{L}(3) \\
& -n(1) n(2,3) \mathrm{M}(1) \cdot \mathrm{L}(2,3) \\
& +2 n(1) n(2) n(3) \mathrm{M}(1) \cdot \mathrm{L}(2) \cdot \mathrm{L}(3)] \cdot\langle\mathbf{E}\rangle+\cdots
\end{aligned}
$$


The general relation is

$$
\begin{aligned}
\langle\mathbf{P}\rangle= & \sum_{s=1}^{\infty} \frac{1}{(s-1) !} \int \cdots \int d \mathbf{R}_{1} \ldots d \mathbf{R}_{s} \sum_{(B)}(-1)^{k-1} n\left(B_{1}\right) \mathrm{M}\left(B_{1}\right) \\
& \cdot n\left(B_{2}\right) \mathrm{L}\left(B_{2}\right) \ldots n\left(B_{k}\right) \mathrm{L}\left(B_{k}\right) \cdot\langle\mathbf{E}\rangle
\end{aligned}
$$

where in the $s$ th term the sum $\sum_{(B)}$ in the integrand is over all ordered partitions of the labels $1, \ldots, s$ into disjoint subsets with the condition that the label 1 be in the first subset. Within the sum, $k=k(B)$ is the number of subsets in the partition $(B)$, and $B_{1}$ is the first subset, $B_{2}$ the second, ..., and $B_{k}$ the $k$ th. We represent a partition into $k$ disjoint (i.e., with no label in common) subsets by $(B)=\left(B_{1}\left|B_{2}\right| \cdots \mid B_{k}\right)$ with slashes indicating the partitioning. Thus, for example, within the three-inclusion integral of (3.16) the second term corresponds to the two partitions $(1,2 \mid 3)$ and $(1,3 \mid 2)$ while the last term corresponds to (1|2|3) and (1|3|2). Note that the ordering of the partition refers to the order of the subsets, within a subset the labels other than the label 1 are not ordered, although we generally write them in ascending order as a convention. The upper limit in the first summation in (3.17) is infinite; for a finite number $N$ of inclusions the partial distribution function $n(1, \ldots, s)$ defined by $(2.8)$ vanishes for $s>N$.

Comparing (3.17) with (2.15) we have a cluster expansion for the electric susceptibility operator $X$, ordered according to the number of particles involved in each term,

$$
\mathrm{X}=\sum_{s=1}^{\infty} \frac{1}{(s-1) !} \mathrm{X}_{s}
$$

where ${ }^{4}$

$$
\mathrm{X}_{s}=\int \cdots \int d \mathbf{R}_{1} \ldots d \mathbf{R}_{s} \sum_{(B)}(-1)^{k-1} n\left(B_{1}\right) \mathrm{M}\left(B_{1}\right) \ldots n\left(B_{k}\right) \mathrm{L}\left(B_{k}\right)
$$

We conclude this section with a number of comments on the expan$\operatorname{sion}(3.18)$.

(1) The expansion is according to the number of inclusions. However, when the density $n$ of inclusions is small we expect in general that $n(1, \ldots, r) \approx n^{r}$, so $\mathrm{X}_{s} \propto n^{s}$. In this sense (3.18) may be viewed as a density expansion.

(2) So far our results are exact for any finite number $N$ of inclusions. In order to justify a statistical treatment we must envisage the thermodynamic limit, in which $N$ and the volume $\Omega$ of the sample grow beyond

\footnotetext{
${ }^{4}$ We remark that if the integrals in the sth terms of (3.8) and (3.12) are considered as generalized moments of the $L(1, \ldots, s)$ and $M(1 ; \ldots s)$ operators then $X_{s}$ is a generalized ordered cumulant, in accordance with the definition by van Kampen. ${ }^{(24,25)}$
} 
bounds such that

$$
n=\lim _{\substack{N \rightarrow \infty \\ \Omega \rightarrow \infty}}(N / \Omega)
$$

is the (constant) density of inclusions. One expects that in this limit the susceptibility operator $X$, or, equivalently, the relation (3.17), exists and is independent of the shape of $\Omega$. This is in accord with our intuition, and the well-accepted notions of macroscopic electrostatics, that the relations between the average fields must be localized. On the other hand, it follows from these same macroscopic considerations that the relations (3.8) and (3.12) between the average fields and the applied field $\mathbf{E}_{0}$ do depend upon the shape of the confining volume $\Omega$. Consider, for example, a volume $\Omega$ which is ellipsoidal in shape and an applied electric field $\mathbf{E}_{0}$ which is uniform (supplied by a charge distribution $\rho_{0}$ outside $\Omega$ ). From macroscopic electrostatics it is known that the average polarization $\langle\mathbf{P}\rangle$ and the average electric field $\langle\mathbf{E}\rangle$ inside the ellipsoid will also be uniform and proportional to $\mathbf{E}_{0}$, but the proportionality is determined by the depolarization tensor for the ellipsoid and is therefore shape dependent. ${ }^{(26)}$ The shape dependence of the relations between the average fields and the applied field is reflected in the conditional convergence of the integrals appearing in the sth term of (3.8) and (3.12): the integrands do not fall off sufficiently fast at large separations of the inclusion centers so that the shape of the bounding surface may be neglected. The expected shape independence of the susceptibility relation should in turn be reflected in the absolute convergence of the integrals in $X_{s}$. It is one of our main objects to provide a careful proof of this fact, which we do in the following sections.

(3) The term $s=1$ in the expansion (3.18) is familiar. If we consider the spatially uniform case, with uniform average field $\langle\mathbf{E}\rangle$ and a uniform density $n$ of inclusions, then we can write

$$
\begin{aligned}
\mathbf{X}_{1} \cdot\langle\mathbf{E}\rangle & =\int d \mathbf{R}_{1} n(1) \mathrm{M}(1) \cdot\langle\mathbf{E}\rangle \\
& =n \int d \mathbf{r} \mathrm{M}(1) \cdot\langle\mathbf{E}\rangle
\end{aligned}
$$

where in the first line the integral is over the position of the inclusion center $\mathbf{R}_{1}$ with fixed field point $\mathbf{r}$ and in the second line the integral is over the field point with fixed position of the inclusion center. But from the definitions (3.9) of $M(1)$ and (2.4) of $K$, we see that $M(1) \cdot\langle\mathbf{E}\rangle$ is the polarization (dipole moment per unit volume) induced in the inclusion placed in field $\langle\mathbf{E}\rangle$. The integral in the second line is therefore the induced dipole moment in the inclusion, which is customarily written as the dipole polarizability $\alpha$ times $\langle\mathbf{E}\rangle$. Hence, in this spatially uniform case,

$$
\mathrm{X}_{1} \cdot\langle\mathbf{E}\rangle=n \alpha\langle\mathbf{E}\rangle
$$


or, if the density $n$ of inclusions is low enough so that the series (3.18) can be approximated by its first term,

$$
\langle\mathbf{P}\rangle \approx n \alpha\langle\mathbf{E}\rangle
$$

which is the familiar relation between polarization and electric field in a medium with a (low) density $n$ of inclusions of polarizability $\alpha$. In the following publication we will use the series (3.18) to compute corrections to (3.23) in the form of an expansion in powers of the density of inclusions.

(4) It might be useful to point out the formal structural similarity between the series (3.8) and (3.12) for the average electric field and the average polarization and the familiar expansions of the pressure and density of a nonideal gas in powers of the fugacity. ${ }^{(27)}$ The elimination of the applied field corresponds in the gas case to the elimination of the fugacity to obtain the virial expansion of the pressure in powers of the density, which is similar in structure to the series (3.17) for the susceptibility relation. In the gas case the coefficients of the fugacity series are integrals over connected cluster functions, for which convergence is not a problem since one always assumes the intermolecular potential falls off faster than the inverse cube of the distance for large separations. But ours is just this marginal case (dipole electric fields fall off as the inverse cube of the distance) which is the reason for the problem of conditional convergence of the integrals in (3.8) and (3.12). Again in the gas case, the coefficients of the virial series are integrals over irreducible cluster functions, with improved convergence. In our case a corresponding irreducible cluster property of the integrand in (3.19) leads to the absolute convergence of the integral.

\section{BINARY COLLISION EXPANSION}

In this section we derive expansions for the operator $K(\mathscr{\tau})$ and the cluster operators $L(\Re)$ and $M(\Re)$ in terms of the repeated action of the operator $\mathrm{M}(1)$ for a single inclusion. Thus the many-inclusion operators are expressed in terms of the (repeated) effects of single isolated inclusions. We will use these expansions to make asymptotic estimates of the cluster operators corresponding to configurations with widely separated inclusions. More important, we will use these expansions in Section 6 to characterize the irreducible cluster property leading to the absolute convergence of the integral (3.19).

We begin with the expression (2.7) for the induced polarization in the presence of a fixed configuration of inclusions. Using (3.3) we can write

$$
\mathbf{P}(\mathscr{T})=\sum_{j \in \mathscr{\Re}} \chi(j) \mathrm{K}(\mathscr{T}) \cdot \mathbf{E}_{0}
$$

where $\Re$ is the set of inclusion labels and the sum is over all the labels in 
$\Re$. This polarization is the source for the induced electric field due to the presence of the inclusions. Since the total electric field $\mathbf{E}(\Re)$ is the sum of the applied field and the induced field, we can write

$$
\mathbf{E}(\Re)=\mathbf{E}_{0}+G_{0} \cdot \mathbf{P}(\Re)
$$

where $\epsilon_{0}$ is Green's function for the electric field due to a given polarization field in a uniform medium with dielectric constant $\epsilon_{1}$. The explicit form for $G_{0}$ acting on a given vector field $V(\mathbf{r})$ is

$$
\left[\epsilon_{0} \cdot \mathbf{V}\right](\mathbf{r})=-\frac{4 \pi}{3 \epsilon_{1}} \mathbf{V}(\mathbf{r})+\int_{\delta} d \mathbf{r}^{\prime} \frac{3\left(\mathbf{r}-\mathbf{r}^{\prime}\right) \cdot \mathbf{V}\left(\mathbf{r}^{\prime}\right)\left(\mathbf{r}-\mathbf{r}^{\prime}\right)-\left(\mathbf{r}-\mathbf{r}^{\prime}\right)^{2} \mathbf{V}\left(\mathbf{r}^{\prime}\right)}{\epsilon_{1}\left|\mathbf{r}-\mathbf{r}^{\prime}\right|^{5}}
$$

where the subscript $\delta$ on the integral indicates that the integral is carried out with the exclusion of an infinitesimally small sphere centered at $\mathbf{r}$. But the definition (2.4) of the operator $\mathrm{K}$ can be written

$$
\mathbf{E}(\mathscr{T})=\mathrm{K}(\mathscr{T}) \cdot \mathbf{E}_{0}
$$

Putting this and (4.1) in (4.2), and using the fact that the resulting equation must be true for arbitrary $\mathbf{E}_{0}(\mathbf{r})$, we find

$$
\mathrm{K}(\mathscr{N})=1+G_{0} \cdot \sum_{j \in \mathscr{N}} \chi(j) \mathrm{K}(\mathscr{T})
$$

This is an integral equation for the operator $\mathrm{K}$. It will be the basis for our discussion in this section. We should emphasize that it is only correct for inclusions that do not overlap.

If we solve the equation (4.5) by iteration we get

$$
\mathrm{K}(\Re)=\sum_{l=0}^{\infty} \sum_{[j]} \prod_{i=1}^{l} \epsilon_{0} \chi\left(j_{i}\right)
$$

where the second sum is over all sequences $[j]=\left[j_{1} j_{2} \ldots j_{l}\right]$ of $l$ labels (counting repetitions) selected from the set $\pi$; the term $l=0$ corresponds to the identity. For example, if $\Re$ consists of the two labels 1 and 2 then, indicating the terms in (4.6) by the corresponding sequences,

$$
\begin{aligned}
\mathrm{K}(1,2)=1 & +([1]+[2])+([11]+[12]+[21]+[22]) \\
+ & ([111]+[112]+[121]+[211]+[122] \\
& +[212]+[221]+[222])+\cdots
\end{aligned}
$$

The case where the set $\Re$ consists of a single label, say 1, corresponds to the sum over sequences in which the label is repeated any number of times. If we then multiply by $\chi(1)$, we get the corresponding rooted cluster 
operator defined in (3.9). Thus

$$
\begin{aligned}
M(1) & =\chi(1) \sum_{l=0}^{\infty}\left[G_{0} \chi(1)\right]^{l} \\
& =\chi(1)+\chi(1) G_{0} \chi(1)+\cdots
\end{aligned}
$$

This result allows us to sum out all the terms in the general expression (4.6) corresponding to sequences in which a label is repeated more than once in succession, expressing the sum in terms of the single-inclusion cluster operator $\mathrm{M}(j)$. This leads to

$$
\mathrm{K}(\Re)=1+\sum_{l=1}^{\infty} \sum_{[j]}^{\prime} \prod_{i=1}^{l} \mathrm{G}_{0} \cdot \mathrm{M}\left(j_{i}\right)
$$

where the second sum is over all sequences $[j]$ of $l$ labels (counting repetitions) selected from the set $\pi$ with the condition, indicated by the prime, that no label be repeated in succession. For example, if $\Re$ consists of the two labels 1 and 2 then, indicating the terms in (4.8) by the corresponding sequences,

$$
\mathrm{K}(1,2)=1+([1]+[2])+([12]+[21])+([121]+[212])+\cdots
$$

Comparing this example with the one following (4.6) we see that the number of terms is significantly reduced. What is more important, the expansion (4.6) is a (weak-coupling) perturbation expansion in powers of $\chi$, useful only when the dielectric constant within the inclusions is close to that in the medium outside, while the expansion (4.8) is a (strong-coupling) expansion in powers of $M\left(j_{i}\right)$. Since the operator $M(1)$ acting on a given field produces exactly the polarization induced in a single isolated inclusion placed in the field with its center at $\mathbf{R}_{1}$, this expansion expresses the field in the presence of $N$ inclusions in terms of the effects of single isolated inclusions. We call the expansion (4.8) the binary collision ${ }^{5}$ expansion of $\mathrm{K}(\Re)$ because of its formal similarity with the corresponding expansion in statistical mechanics. ${ }^{(28,29)}$ It is also called the multiple scattering expansion $^{(30)}$ or the single-site expansion. ${ }^{(31)}$

To get the binary collision expansion of the cluster operator $L$ we put the expansion (4.8) of $\mathrm{K}$ in the expression (3.7), which gives

$$
\mathrm{L}(\Re)=\sum_{l=N}^{\infty} \sum_{[j]}^{\prime \prime} \prod_{i=1}^{l} \epsilon_{0} \cdot \mathrm{M}\left(j_{i}\right)
$$

\footnotetext{
${ }^{5}$ In the original binary collision expansion the solution of the $N$-body problem is expressed in terms of the exact solution for isolated pairs of particles. In our case the "pair" corresponds to the inclusion and the electric field.
} 
where the second sum is over all sequences $[j]$ of $l$ labels selected from the set $\Re$ with the conditions, indicated by the double prime, that no label be repeated in succession and that every label in $\Re$ occur at least once in the sequence. For example,

$$
\begin{aligned}
L(1,2,3)=( & {[123]+[132]+[213]+[231]+[312]+[321]) } \\
+ & ([1213]+[1231]+[1321]+[1312]+[1232]+[1323] \\
& +[2123]+[2132]+[2312]+[2321] \\
& +[2131]+[2313]+[3231]+[3213]+[3123] \\
& +[3132]+[3212]+[3121])+\cdots
\end{aligned}
$$

where again we have indicated only the sequences to which the terms correspond. To get this result we use (4.8) in (3.7) to yield

$$
\mathrm{L}(\Re)=\sum_{\Re \in \Re}(-1)^{N-M} \sum_{l=0}^{\infty} \sum_{[j]: \Re \prod_{i=1}^{\prime}}^{l} \prod_{i=1}^{l} \epsilon_{0} \cdot \mathrm{M}\left(j_{i}\right)
$$

where the first sum is over subsets $\Re$ of the given set $\Re$ and the third sum is over sequences $[j]$ of labels selected from $\Re$ (indicated by $[j]: \mathfrak{T}$ ) with the conditions that there be $l$ labels in the sequence and that no label be repeated in succession. These sums can be rearranged to write

$$
L(\Re)=\sum_{l=0}^{\infty} \sum_{[j]: \Re}^{\prime} \prod_{i=1}^{l} \epsilon_{0} \cdot M\left(j_{i}\right) \sum_{g \subset \Re \subset \Re}(-1)^{N-M}
$$

where now the second sum is over sequences of labels selected from the given set $\mathscr{r}$ and the third sum is over those subsets $\mathscr{R}$ of $\mathscr{r}$ which themselves have the subset $\xi$, the set of labels which occur in the sequence $[j]$. This last sum is simple to evaluate if we note that the number of sets $q$ which contain exactly $M$ labels and which are subsets of $\pi$ and have subset $f$ is the binomial coefficient $\left(\begin{array}{c}N-J \\ M-J\end{array}\right)$, where $N$ and $J$ are, respectively, the number of labels in $\Re$ and $g$. Hence

$$
\sum_{g \subset \mathscr{N} \subset \mathcal{X}}(-1)^{N-M}=\sum_{M=J}^{N}\left(\begin{array}{l}
N-J \\
M-J
\end{array}\right)(-1)^{N-M}= \begin{cases}0, & J<N \\
1, & J=N\end{cases}
$$

Using this in (4.11) we have the result (4.9).

The binary collision expansion of the rooted cluster operator $M$ is obtained by minor modification of the above arguments. The result can be written

$$
\mathrm{M}(\Re)=\sum_{l=N}^{\infty} \sum_{[j]}^{\prime \prime \prime} \mathrm{M}\left(j_{1}\right) \cdot \prod_{i=2}^{l} \epsilon_{0} \cdot \mathrm{M}\left(j_{i}\right)
$$

where $N$ is the number of labels in the set $\Re$, which must contain the label 
1 , and the second sum is over all sequences $[j]$ of $l$ labels selected from the set $\pi$ with the conditions, indicated by the triple prime, that no label be repeated in succession, that every label in $\pi$ occur at least once, and that the label 1 be the first label in the sequence (i.e., $j_{1}=1$ ). For example, indicating terms by their corresponding sequences,

$$
\begin{aligned}
& M(1 ; 2,3)=([123]+[132])+([1213]+[1231]+[1321] \\
&+[1312]+[1232]+[1323])+\cdots
\end{aligned}
$$

A property of the operator $M(1)$ which is important for determining the behavior of the cluster operators for large separations of the inclusions is that it is localized. To see the meaning of this we use an expanded notation in which $\mathrm{M}(1)$ is written as an integral operator acting on an electrostatic field $\mathbf{E}(\mathbf{r})$,

$$
[\mathrm{M}(1) \cdot \mathbf{E}]_{j}(\mathbf{r})=\int d \mathbf{r}^{\prime} M_{j k}\left(1 ; \mathbf{r}, \mathbf{r}^{\prime}\right) E_{k}\left(\mathbf{r}^{\prime}\right)
$$

where repeated indices are summed over the three spatial directions. The localized property of $M(1)$ is that

$$
M_{j k}\left(1 ; \mathbf{r}, \mathbf{r}^{\prime}\right)=0, \quad \text { if } \quad\left|\mathbf{r}-\mathbf{R}_{\mathrm{l}}\right|>a \text { or }\left|\mathbf{r}^{\prime}-\mathbf{R}_{\mathrm{I}}\right|>a
$$

which follows from (4.7) and the fact that $\chi(1)$ vanishes outside the inclusion. Recalling that $M(1) \cdot \mathbf{E}_{0}$ is the induced polarization in the presence of a single inclusion placed in the external field $\mathbf{E}_{0}(\mathbf{r})$, this localized property is just the intuitively obvious property that the induced polarization vanishes except within the inclusion and depends only upon the field $\mathbf{E}_{0}$ within the inclusion. Note also that, because each term in the expansion (4.13) has a factor $M(1)$ to the left, the rooted cluster operators all have the property that they vanish unless the field point is within the inclusion centered at $\mathbf{R}_{1}$. More explicitly, using an expanded notation as in (4.13),

$$
\mathrm{M}\left(1 ; 2, \ldots, s ; \mathbf{r}, \mathbf{r}^{\prime}\right)=0, \quad \text { if }\left|\mathbf{r}-\mathbf{R}_{1}\right|>a
$$

Again, this is an expression of the intuitively obvious fact that the induced polarization vanishes outside the inclusion.

This localized property of $M(1)$ allows us to visualize the term corresponding to a given sequence in the binary collision expansion. The labels in the sequence indicate a localization of the field point at the corresponding inclusion and between successive labels there is a factor $G_{0}$ which "propagates" the field from one inclusion to the other. With this picture we can by inspection of a sequence see the behavior of the corresponding term for large separations of the inclusions. Thus, for example, if the labels 1 and 2 occur successively in a sequence, and the centers $\mathbf{R}_{1}$ and $\mathbf{R}_{2}$ are widely separated, the localized property (4.16) and the form (4.3) of $G_{0}$ tell us that there will be a factor of order $R_{12}^{-3}$ in the corresponding term. 
The asymptotic behavior of the cluster operators for large separations of the inclusions can be seen with the help of this picture. Thus if we consider a configuration in which the inclusions are in two widely separated groups, corresponding to a partition of the labels into two disjoint sets $B_{1}$ and $B_{2}$, then the sum (4.9) for $L$ will be dominated by the sequences which involve only a single switch from one group to the other. Thus

$$
\mathrm{L}\left(B_{1}, B_{2}\right) \sim \mathrm{L}\left(B_{1}\right) \cdot \mathrm{L}\left(B_{2}\right)+\mathrm{L}\left(B_{2}\right) \cdot \mathrm{L}\left(B_{1}\right)+\mathcal{\theta}\left(R_{\min }^{-6}\right)
$$

where $R_{\min }$ is the minimum distance between the centers whose labels are in $B_{1}$ and those whose labels are in $B_{2}$. Generalizing to a configuration with $k$ widely separated groups, corresponding to a partition of the labels into $k$ disjoint subsets we have

$$
\mathrm{L}\left(B_{1}, \ldots, B_{k}\right) \sim \sum_{\mathscr{P}} \mathrm{L}\left(B_{1}\right) \ldots \mathrm{L}\left(B_{k}\right)+\vartheta\left(R_{\min }^{-3 k}\right)
$$

where the sum is over all permutations of the order of the cluster operators and $R_{\min }$ is the minimum distance between groups. Of course, by the same arguments, we know that the leading term in (4.18) is of order $R_{\min }^{-3(k-1)}$. The corresponding result for the rooted cluster operator is obtained in the same way. We consider a configuration with $k$ widely separated groups, a group containing the inclusion centered at $\mathbf{R}_{1}$ and $k-1$ other groups. With this configuration is associated a partition of the labels into $k$ disjoint subsets with $B_{1}$ the subset of labels corresponding to the inclusions near $\mathbf{R}_{1}$ and $B_{2}, \ldots, B_{k}$ the subsets of labels corresponding to the $k-1$ other groups. Then

$$
\mathrm{M}\left(B_{1}, B_{2}, \ldots, B_{k}\right) \sim \mathrm{M}\left(B_{1}\right) \cdot \sum_{\mathscr{P}} \mathrm{L}\left(B_{2}\right) \ldots \mathrm{L}\left(B_{k}\right)+\mathcal{O}\left(R_{\min }^{-3 k}\right)
$$

where the sum is over all permutations of the order of the cluster operators and $R_{\min }$ is the minimum distance between groups.

\section{REARRANGEMENT OF THE TERMS}

In the next section we will give a proof of the absolute convergence of the cluster integrals $X_{s}$ appearing in the expansion (3.18) for the electric susceptibility operator. Here we give some preliminary discussion of the form of these integrals, first introducing a shorthand notation for the terms in the integrands and then, after some general remarks, introducing a rearrangement of the terms in the integrand which will facilitate the proof.

The terms in the integrand of $\mathbf{X}_{s}$ are associated with ordered partitions of the labels. Explicitly, for the case $s=2$ the expression (3.19) for $\mathrm{X}_{s}$ 
becomes

$$
\mathrm{X}_{2}=\iint d \mathbf{R}_{1} d \mathbf{R}_{2}[n(1,2) \underset{(1,2)}{\mathrm{M}}(1 ; 2)-n(1) n(2) \underset{(1 \mid 2)}{\mathrm{M}}(1) \cdot L(2)]
$$

where we have indicated beneath each term the partition to which it corresponds. In the same way we write for $s=3$

$$
\begin{aligned}
\mathrm{X}_{3}=\iiint d \mathbf{R}_{1} d \mathbf{R}_{2} d \mathbf{R}_{3}[ & n(1,2,3) \underset{(1,2,3)}{\mathrm{M}}(1 ; 2,3)-n(1,2) n(3) \underset{(1,2 \mid 3)}{\mathrm{M}}(1 ; 2) \\
& \cdot \mathrm{L}(3)-n(1) n(2,3) \underset{(1 \mid 2,3)}{\mathrm{M}}(1) \cdot \mathrm{L}(2,3) \\
& -n(1,3) n(2) \underset{(1,3 \mid 2)}{\mathrm{M}}(1 ; 3) \cdot \mathrm{L}(2) \\
& +n(1) n(2) n(3) \underset{(1|2| 3)}{\mathrm{M}}(1) \cdot \mathrm{L}(2) \cdot \mathrm{L}(3) \\
& +n(1) n(2) n(3) \underset{(1|3| 2)}{\mathrm{M}}(1) \cdot \mathrm{L}(3) \cdot \mathrm{L}(2)]
\end{aligned}
$$

In general the integrand of $\mathrm{X}_{s}$ is the sum of terms corresponding to the partitions of the labels into ordered disjoint subsets with the condition that the label 1 be in the first subset.

The term associated with a given partition is a product of factors of cluster operators and partial distribution functions, each of which can be associated with the partition. For the cluster operators we have the asymptotic estimates based on the results of the previous section. The corresponding estimates for the partial distribution functions depend upon the form of the distribution $W(1, \ldots, N)$. Here we assume this distribution is such that the partial distribution functions have the product property: for a configuration with $k$ widely separated groups, corresponding to a partition of the labels into $k$ disjoint subsets $B_{1}, B_{2}, \ldots, B_{k}$, then

$$
n\left(B_{1}\left|B_{2}\right| \ldots \mid B_{k}\right) \sim n\left(B_{1}\right) n\left(B_{2}\right) \ldots n\left(B_{k}\right)
$$

We will assume the corrections to this asymptotic estimate are negligibly small. This is the familiar product property of the partial distribution functions of a nonideal gas. ${ }^{(27)}$

As we remarked at the end of Section 3, in analogy with the virial expansion for the nonideal gas, we expect that the integrand in $\mathrm{X}_{s}$ should in some sense be irreducible, with improved convergence. To see how this comes about consider first the case $s=2$. Recall first that, because of the general property (4.15), the integration over $\mathbf{R}_{1}$ is confined to within a 
sphere of radius $a$ about the field point, which is considered as fixed. We need therefore consider only the behavior of the $\mathbf{R}_{2}$ integration when $\mathbf{R}_{2}$ is far from $\mathbf{R}_{1}$. In this case, using the asymptotic estimates (4.19) and (5.3), the integrand of $(5.1)$ becomes

$$
(1,2)-(1 \mid 2) \sim n(1) n(2) \mathrm{M}(1) \cdot \mathrm{L}(2)-n(1) n(2) \mathrm{M}(1) \cdot \mathrm{L}(2)+\vartheta\left(R_{12}^{-6}\right)
$$

so the leading terms cancel and the integrand is $\theta\left(R_{12}^{-6}\right)$, which is absolutely convergent. Consider next the case $s=3$ and the configuration in which $\mathbf{R}_{3}$ is far from $\mathbf{R}_{2}$ which is close to $\mathbf{R}_{1}$ (which in turn is within a radius $a$ of the field point). Using the asymptotic estimates (4.18), (4.19), and (5.3), the integrand of (5.2) becomes

$$
\begin{gathered}
(1,2,3)-(1,2 \mid 3)-(1 \mid 2,3)-(1,3 \mid 2)+(1|2| 3)+(1|3| 2) \\
\sim-n(1) n(2) n(3) \mathrm{M}(1) \cdot \mathrm{L}(3) \cdot \mathrm{L}(2)+\vartheta\left(R_{\min }^{-6}\right)
\end{gathered}
$$

Thus leading terms in the asymptotic estimate do not cancel completely. On the other hand, this does not spoil the absolute convergence since the remainder is itself $\mathcal{O}\left(R_{\min }^{-6}\right)$. This we see since the binary collision expansion of the operator in the remainder consists of a single term corresponding to the sequence [132],

$$
M(1) \cdot L(3) \cdot L(2)=M(1) \cdot G_{0} \cdot M(3) \cdot G_{0} \cdot M(2)
$$

and for configurations in which $\mathbf{R}_{3}$ is far from $\mathbf{R}_{1}$ and $\mathbf{R}_{\mathbf{2}}$ there will be a factor $\mathcal{O}\left(R_{23}^{-3}\right)$ from the $G_{0}$ between $M(3)$ and $M(2)$ and a factor $\mathcal{O}\left(R_{13}^{-3}\right)$ from the $G_{0}$ between $M(1)$ and $M(3)$. In the same way we can consider the other asymptotic configurations of the integrand of (5.2), that in which $\mathbf{R}_{2}$ is far from $\mathbf{R}_{3}$ which is close to $\mathbf{R}_{\mathbf{1}}$ and that in which $\mathbf{R}_{2}$ is far from $\mathbf{R}_{3}$ and both are far from $\mathbf{R}_{1}$, and show that in each case there is absolute convergence. These examples, which amount to a proof of the absolute convergence of the integrals in $X_{2}$ and $X_{3}$, show the idea of the proof for the general case. However, they also show that in the general case we will need to have a systematic scheme for handling the cancellations and discussing the remainder. This we introduce in the rest of this section.

Our scheme for rearranging the terms in the integrand of $\mathrm{X}_{s}$ is based on the lattice of ordered partitions of the set of labels $1,2, \ldots, s$. This lattice is an arrangement of the partitions in rows according to the number of disjoint subsets. The first (top) row contains the partition into one subset, i.e., the set of labels itself; the second row contains the partitions into two subsets, etc. In the lattice each partition is associated with those partitions in the row above which are obtained by merging adjoining subsets, i.e., 
removing a slash. Thus for $s=2$, the lattice consists of two rows,

and for $s=3$,

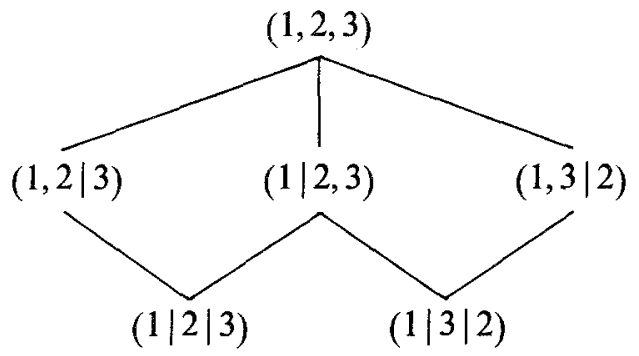

This arrangement results in a partial ordering of the partitions (denoted by $\leqslant)$ with $(B) \leqslant\left(B^{\prime}\right)$ if the partition $\left(B^{\prime}\right)$ is either the same as the partition $(B)$ or can be obtained from $(B)$ by removing one or more slashes. For example, in the lattice for $s=3,(1|2| 3) \leqslant(1 \mid 2,3)$ and $(1|2| 3) \leqslant(1,2,3)$ but there is no ordering between $(1|2| 3)$ and $(1,3 \mid 2)$. A key identity which we will need in our later discussion is the following. For any two partitions $(B)$ and $\left(B^{\prime}\right)$ such that $(B) \leqslant\left(B^{\prime}\right)$,

$$
\sum_{(B) \leqslant\left(B^{\prime \prime}\right) \leqslant\left(B^{\prime}\right)}(-1)^{k^{\prime \prime}-k}= \begin{cases}0, & (B) \neq\left(B^{\prime}\right) \\ 1, & (B)=\left(B^{\prime}\right)\end{cases}
$$

where the sum is over the partitions ( $\left.B^{\prime \prime}\right)$ which can be obtained from $(B)$ by removing slashes and from which $\left(B^{\prime}\right)$ can be obtained by removing slashes, and where $k=k(B)$ and $k^{\prime \prime}=k\left(B^{\prime \prime}\right)$ are, respectively, the row numbers in which $(B)$ and $\left(B^{\prime \prime}\right)$ lie. The proof follows from the fact that the number of partitions in the sum which are in the $k^{\prime \prime}$ th row is $\left(\begin{array}{c}k^{\prime}-k \\ k^{\prime \prime}-k\end{array}\right)$, the number of ways one can select $k^{\prime \prime}-k$ of the $k^{\prime}-k$ slashes which must be removed from $(B)$ to get $\left(B^{\prime}\right)$. The sum is therefore

$$
\sum_{k^{\prime \prime}=k}^{k^{\prime}}\left(\begin{array}{c}
k^{\prime}-k \\
k^{\prime \prime}-k
\end{array}\right)(-1)^{k^{\prime \prime}-k}=(1-1)^{k^{\prime}-k}= \begin{cases}0, & k^{\prime} \neq k \\
1, & k^{\prime}=k\end{cases}
$$

which is equivalent to (5.7).

The strategy of our rearrangement is perhaps best explained by considering first the cases $s=2$ and $s=3$. The integrand of (5.1) can be written in 
the form

$$
n(1,2)[\underset{(1,2)}{\mathrm{M}}(1 ; 2)-\mathrm{M}(1) \cdot \mathrm{L}(2)]+[n(1,2)-n(1) \underset{(1 \mid 2)}{n}(2)] \mathrm{M}(1) \cdot \mathrm{L}(2)
$$

Note that each term in the rearrangement is itself associated with a partition of the labels. Now, however, the term associated with a given partition leads to an absolutely convergent integral, that associated with the the partition $(1,2)$ on account of the asymptotic estimate (4.19) of the cluster operators and that associated with the partition $(1 \mid 2)$ on account of the product property (5.3) of the partial distribution functions. Before stating the general rule, we exhibit the rearrangement for the case $s=3$, writing the integrand of (5.2) in the form

$$
\begin{aligned}
& n(1,2,3)[M(1 ; 2,3)-M(1) \cdot \underset{(1,2,3)}{L}(2,3)-M(1 ; 2) \cdot L(3)-M(1 ; 3) \cdot L(2) \\
& +M(1) \cdot L(2) \cdot L(3)+M(1) \cdot L(3) \cdot L(2)] \\
& +[n(1,2,3)-n(1,2) n(3)][\underset{(1,2 \mid 3)}{M}(1 ; 2) \cdot L(3)-M(1) \cdot L(2) \cdot L(3)] \\
& +[n(1,2,3)-n(1) n(2,3)] \\
& \times[\underset{(1 / 2,3)}{M}(1) \cdot L(2,3)-M(1) \cdot L(2) \cdot L(3)-M(1) \cdot L(3) \cdot L(2)] \\
& +[n(1,2,3)-n(1,3) n(2)][\underset{(1,3 \mid 2)}{M}(1 ; 3) \cdot L(2)-M(1) \cdot L(3) \cdot L(2)] \\
& +[n(1,2,3)-n(1) n(2,3)-n(1,2) \underset{(1|2| 3)}{n}(3)+n(1) n(2) n(3)] \\
& \times \mathrm{M}(1) \cdot \mathrm{L}(2) \cdot \mathrm{L}(3)+\left[n(1,2,3)-n(1) n(2,3)-n(1,3)_{(1|3| 2)}^{n}(2)\right. \\
& +n(1) n(2) n(3)] \mathrm{M}(1) \cdot \mathrm{L}(3) \cdot \mathrm{L}(2)
\end{aligned}
$$

Again, the terms in the rearrangement are associated with the same partitions of the labels as in the original form, but now each term leads to an absolutely convergent integral. We can now state the general rule. The integrand in the expression (3.19) for $\mathrm{X}_{s}$ is rewritten in the form

$$
\begin{aligned}
\sum_{(B)}( & -1)^{k-1} n\left(B_{1}\right) \mathrm{M}\left(B_{1}\right) \ldots n\left(B_{k}\right) \mathrm{L}\left(B_{k}\right) \\
& =\sum_{(B)} b\left(B_{1}\left|B_{2}\right| \ldots \mid B_{k}\right) \mathrm{C}\left(B_{1}\left|B_{2}\right| \ldots \mid B_{k}\right)
\end{aligned}
$$

where on both sides the sum is over all partitions $(B)=\left(B_{1}\left|B_{2}\right| \ldots \mid B_{k}\right)$ in 
the lattice of ordered partitions of $s$ labels. The summand on the right is a product of a block distribution function $b\left(B_{1}\left|B_{2}\right| \ldots \mid B_{k}\right)$ and a chain operator $\mathrm{C}\left(B_{1}\left|B_{2}\right| \ldots \mid B_{k}\right)$. The block distribution function is the sum of products of partial distribution functions:

$$
b\left(B_{1}\left|B_{2}\right| \ldots \mid B_{k}\right)=\sum_{\left(B^{\prime}\right) \geqslant(B)}(-1)^{k^{\prime}-1} n\left(B_{1}^{\prime}\right) n\left(B_{2}^{\prime}\right) \ldots n\left(B_{k^{\prime}}^{\prime}\right)
$$

where the sum is over the given partition together with those partitions in all the rows above which can be obtained from it by removing slashes and $k^{\prime}=k\left(B^{\prime}\right)$ is the row number of $\left(B^{\prime}\right)$. Similarly the chain operator is the sum

$$
\mathrm{C}\left(B_{1}\left|B_{2}\right| \ldots \mid B_{k}\right)=\sum_{\left(B^{\prime}\right) \leqslant(B)}(-1)^{k-k^{\prime}} \mathrm{M}\left(B_{1}^{\prime}\right) \cdot \mathrm{L}\left(B_{2}^{\prime}\right) \ldots \mathrm{L}\left(B_{k^{\prime}}^{\prime}\right)
$$

where the sum is over the given partition together with those in all the rows below from which it can be obtained by removing slashes. The proof of the general rule (5.11) follows from the identity (5.7) after inserting (5.12) and (5.13) on the right-hand side of (5.11).

Inserting the expansion (5.11) in (3.19) we can write

$$
\mathrm{X}_{s}=\sum_{(B)} \mathrm{X}(B)
$$

where again the sum is over the ordered partitions of the labels $1,2, \ldots, s$ with the condition that the label 1 be in the first subset and where

$$
\mathrm{X}(B)=\int \cdots \int d \mathbf{R}_{1} \ldots d \mathbf{R}_{s} b(B) \mathrm{C}(B)
$$

In the next section we show that each of these integrals is absolutely convergent.

\section{ABSOLUTE CONVERGENCE}

The integrand of (5.15) is the product of a block distribution function $b(B)$ and a chain operator $\mathrm{C}(B)$, each associated with the partition $(B)=\left(B_{1}\left|B_{2}\right| \ldots \mid B_{k}\right)$. To demonstrate absolute convergence of the integral we show that for widely separated configurations the integrand vanishes sufficiently rapidly, either because of the vanishing of $b(B)$ or of $C(B)$.

First we assert that the block distribution function $b(B)=$ $b\left(B_{1}\left|B_{2}\right| \ldots \mid B_{k}\right)$ vanishes for any configuration in which the coordinates associated with the labels to the right of a given slash are widely separated from those associated with labels to the left. To see this we note that the sum (5.12) defining $b(B)$ is over the partitions in the lattice which can be obtained from $(B)$ by removing slashes. The partitions in the sum can 
therefore be paired, the one having the given slash, the other in the row above obtained by removing the slash. For the separated configuration the terms corresponding to each pair cancel, since they have opposite signs and, on account of the product property (5.3), the same product of partial distribution functions appears in each. As an illustration of this result, $b(1|2| 3)$ vanishes when 2 and 3 are near but far from 1 or when 1 and 2 are near but far from 3; it does not vanish, however, when 1 and 3 are near but far from 2.

We must now show that the chain operator $\mathrm{C}(B)=\mathrm{C}\left(B_{1}\left|B_{2}\right| \ldots \mid B_{k}\right)$ vanishes sufficiently rapidly for those configurations in which coordinates corresponding to labels within one of the subsets $B_{1}, B_{2}, \ldots, B_{k}$ are widely separated. Thus $\mathrm{C}(B)$ will vanish when $b(B)$ does not, leading to the absolute convergence of $X(B)$. To show this we use the binary collision expansion of the chain operator, which we obtain using (4.11) and (4.13) in (5.13). A typical term in (5.13) can then be written

$$
\mathrm{M}\left(B_{1}^{\prime}\right) \cdot \mathrm{L}\left(B_{2}^{\prime}\right) \ldots \mathrm{L}\left(B_{k^{\prime}}^{\prime}\right)=\sum_{l=s}^{\infty} \sum_{[j]}^{*} \mathrm{M}\left(j_{1}\right) \cdot \prod_{i=2}^{l} \mathrm{G}_{0} \cdot \mathrm{M}\left(j_{i}\right)
$$

where the second sum is over sequences $[j]$ of $l$ labels selected from the set $1,2, \ldots, s$ with the conditions, indicated by the asterisk, that no label be repeated in succession, that every label in the set occur at least once, that the label 1 be the first label in the sequence, and that each sequence consist of $k^{\prime}$ disjoint subsequences, the first chosen from the subset $B_{1}^{\prime}$, the second from the subset $B_{2}^{\prime}, \ldots$, the $k$ th from the subset $B_{k^{\prime}}^{\prime}$. When (6.1) is put in the sum (5.13) there will be a cancellation of many terms. To describe this, and also to characterize the terms which survive the calculation we introduce some terminology. A sequence of labels will be called reducible if it consists of two or more disjoint subsequences, that is if it can be written as a sequence of labels chosen from one subset of the labels $1,2, \ldots, s$ followed by a sequence of labels chosen from another disjoint subset. For example, $[1213]=[121][3]$ is reducible while $[1231]$ is not. A sequence which is not reducible will be called irreducible. Clearly the sequences in the expansion (6.1) are in general reducible, while the disjoint subsequences of labels chosen from $B_{1}^{\prime}, B_{2}^{\prime}, \ldots, B_{k^{\prime}}^{\prime}$ may or may not themselves be reducible. However, when (6.1) is inserted in (5.13) we can show that the cancellations lead to the result

$$
\mathrm{C}\left(B_{1}\left|B_{2}\right| \ldots \mid B_{k}\right)=\sum_{l=s}^{\infty} \sum_{[j]}^{\dagger} \mathrm{M}\left(j_{1}\right) \cdot \prod_{i=2}^{l} \epsilon_{0} \cdot \mathrm{M}\left(j_{i}\right)
$$

where the sum is over sequences $[j]$ of $l$ labels with the conditions, indicated by the dagger, that no label be repeated in succession, that every label occur at least once, that the label 1 be the first label in the sequence, 
and that each sequence consist of $k$ disjoint irreducible subsequences, the first chosen from the subset $B_{1}, \ldots$, the last from the subset $B_{k}$. In particular, in the binary collision expansion of the chain operator $\mathrm{C}(1 ; 2, \ldots, s)$ associated with the top partition in the lattice, only irreducible sequences occur. Thus, for example,

$$
\begin{aligned}
C(1 ; 2,3)= & ([1231]+[1321]) \\
+ & ([12321]+[13231]+[12131]+[13121]+[12312] \\
& +[12313]+[13213]+[13212]+[12132] \\
& +[13123])+\cdots
\end{aligned}
$$

where, again, as in Section 4, we indicate the terms by their corresponding sequences.

To demonstrate the result (6.2) we note first that with a given reducible sequence occurring in (6.1) we can associate a unique ordered partition of the labels $1,2, \ldots, s$. We do this by identifying the sets of labels in the successive irreducible subsequences of the given sequence with the successive subsets of the ordered partition. For example, if $s=3$, the reducible sequence [1213] consists of the two successive irreducible subsequences [121] and [3] and hence is associated with the ordered partition (12|3). Now the reducible sequences which occur in the sum (6.1) will in general correspond to a partition $\left(B^{\prime \prime}\right) \leqslant\left(B^{\prime}\right)$ since the constraint on the sum does not rule out the possibility that the $k^{\prime}$ disjoint subsequences are themselves reducible. On the other hand, if we fix our attention on a particular reducible sequence associated with the partition $\left(B^{\prime \prime}\right) \leqslant(B)$, it will occur exactly once in the binary collision expansion (6.1) for each partition in the sum (5.13) such that $\left(B^{\prime \prime}\right) \leqslant\left(B^{\prime}\right) \leqslant(B)$. But, from the identity (5.7), these terms precisely cancel when the sum over $\left(B^{\prime}\right)$ is carried out. The remaining terms are just those given in the expansion (6.2), which is the binary collision expansion of the chain operator.

We see, therefore, that the chain operator is a product of irreducible operators associated with each of the disjoint subsets. More explicitly,

$$
\mathrm{C}\left(B_{1}\left|B_{2}\right| \ldots \mid B_{k}\right)=\mathrm{C}\left(B_{1}\right) \cdot G_{0} \cdot \mathrm{D}\left(B_{2}\right) \ldots G_{0} \cdot \mathrm{D}\left(B_{k}\right)
$$

where $\mathrm{C}\left(B_{1}\right)$ has a binary collision expansion of the form (6.2) but in which only irreducible sequences occur, $\mathrm{D}\left(\boldsymbol{B}_{2}\right)$ is a similar sum over irreducible sequences but with no restriction as to which label is first, etc. The irreducible operators $C$ and $D$ in (6.3) may be regarded as the beads in a chain of beads with the Green's function propagators $G_{0}$ as the links. The irreducible property of the beads means that for any configuration in which there is a wide separation of inclusions corresponding to labels within a bead, there must be at least two factors of $G_{0}$ linking the separated 
inclusions. As a consequence the chain operator will vanish as the inverse sixth power of the separation, leading to absolute convergence. On the other hand, for configurations in which there are wide separations corresponding to the links between the beads the chain operators only vanish as the inverse cube of the separation, but for exactly these configurations the corresponding block distribution function vanishes. Hence the product occurring in the integrand of (5.15) vanishes sufficiently rapidly for all configurations and the integral is absolutely convergent.

\section{EFFECTIVE DIELECTRIC CONSTANT}

In this section our aim is to give an expansion for the effective dielectric constant $\epsilon^{*}$ of the system. We do this by first using the assumption of spatial homogeneity of the distribution of inclusions to obtain an expression for the effective dielectric susceptibility tensor, which relates the average polarization to the average electric field. We then use (2.14) to identify the effective dielectric tensor. Finally, specializing to the case of a uniform applied field and an isotropic as well as homogeneous distribution of inclusions, we obtain the desired expression for $\epsilon^{*}$.

Assuming then that the distribution of inclusions is spatially homogeneous, the susceptibility relation (2.15) will be invariant under spatial translations, i.e., the susceptibility kernel will be of the form

$$
X_{j k}\left(\mathbf{r}, \mathbf{r}^{\prime}\right)=X_{j k}\left(\mathbf{r}-\mathbf{r}^{\prime}\right)
$$

In this case it is appropriate to introduce the spatial Fourier transform of the fields, writing

$$
\begin{aligned}
& \langle\mathbf{E}(\mathbf{r})\rangle=\int d \mathbf{q} \exp (i \mathbf{q} \cdot \mathbf{r})\left\langle\mathbf{E}_{\mathbf{q}}\right\rangle \\
& \langle\mathbf{P}(\mathbf{r})\rangle=\int d \mathbf{q} \exp (i \mathbf{q} \cdot \mathbf{r})\left\langle\mathbf{P}_{\mathbf{q}}\right\rangle
\end{aligned}
$$

etc. Using (7.1) the susceptibility relation (2.15) can then be written

$$
\left\langle\mathbf{P}_{\mathbf{q}}\right\rangle=\boldsymbol{x}^{*}(\mathbf{q}) \cdot\left\langle\mathbf{E}_{\mathbf{q}}\right\rangle
$$

where $\boldsymbol{\chi}^{*}(\mathbf{q})$ is the effective susceptibility tensor. To get an expression for $\chi^{*}(q)$ we first form the Fourier transform of the susceptibility relation (2.15) to write

$$
\left\langle\mathbf{P}_{\mathbf{q}}\right\rangle=\frac{1}{(2 \pi)^{3}} \int d \mathbf{q}^{\prime}\left(\mathbf{q}|\mathbf{X}| \mathbf{q}^{\prime}\right)\left\langle\mathbf{E}_{\mathbf{q}}^{\prime}\right\rangle
$$

where

$$
\left(\mathbf{q}|\mathbf{X}| \mathbf{q}^{\prime}\right) \equiv \int d \mathbf{r} \int d \mathbf{r}^{\prime} \exp (-i \mathbf{q} \cdot \mathbf{r}) X\left(\mathbf{r}, \mathbf{r}^{\prime}\right) \exp \left(i \mathbf{q}^{\prime} \cdot \mathbf{r}^{\prime}\right)
$$


Using the expansion (5.15) in the sum (3.18) for $X$, we can write

$$
\left(\mathbf{q}|\mathbf{X}| \mathbf{q}^{\prime}\right)=\sum_{s=1}^{\infty} \frac{1}{(s-1) !} \sum_{(B)} \int \cdots \int d \mathbf{R}_{\mathbf{1}} \ldots d \mathbf{R}_{s} b(B)\left(\mathbf{q}|\mathbf{C}(B)| \mathbf{q}^{\prime}\right)
$$

Now, the block distribution functions $b(B)$ have the translation invariance property of the underlying distribution of inclusions. Specifically, this means in (7.6) that $b(B)$ depends only upon the coordinates $\mathbf{R}_{2}-\mathbf{R}_{1}, \mathbf{R}_{3}-$ $\mathbf{R}_{1}, \ldots, \mathbf{R}_{s}-\mathbf{R}_{1}$ of the inclusions relative to the coordinate $\mathbf{R}_{1}$. On the other hand, translating the inclusion centered at $\mathbf{R}_{1}$ to the origin, we can write

$$
\left(\mathbf{q}|\mathbf{C}(B)| \mathbf{q}^{\prime}\right)=\exp \left[i\left(\mathbf{q}^{\prime}-\mathbf{q}\right) \cdot \mathbf{R}_{1}\right]\left(\mathbf{q}\left|\{\mathbf{C}(B)\}_{\mathbf{R}_{j} \rightarrow \mathbf{R}_{j}-\mathbf{R}_{1}}\right| \mathbf{q}^{\prime}\right)
$$

where on the right the chain operator depends only upon the coordinates of the inclusions relative to $\mathbf{R}_{1}$. Putting this in (7.6), the $\mathbf{R}_{1}$ integration gives

$$
\int d \mathbf{R}_{1} \exp \left[i\left(\mathbf{q}^{\prime}-\mathbf{q}\right) \cdot \mathbf{R}_{1}\right]=(2 \pi)^{3} \delta\left(\mathbf{q}^{\prime}-\mathbf{q}\right)
$$

and (7.4) takes the form (7.3) with

$$
\chi^{*}(\mathbf{q})=\sum_{s=1}^{\infty} \frac{1}{(s-1) !} \sum_{(B)} \int \cdots \int d \mathbf{R}_{2} \ldots d \mathbf{R}_{s}\{b(B)(\mathbf{q}|\mathrm{C}(B)| \mathbf{q})\}_{\mathbf{R}_{1}=0}
$$

Next, we form the Fourier transform of (2.14) and use (7.3) to write

$$
\left\langle\mathbf{D}_{\mathbf{q}}\right\rangle=\mathbf{\epsilon}^{*}(\mathbf{q}) \cdot\left\langle\mathbf{E}_{\mathbf{q}}\right\rangle
$$

where

$$
\epsilon^{*}(\mathbf{q})=\epsilon_{1} 1+4 \pi \chi^{*}(\mathbf{q})
$$

is the effective dielectric tensor. If, as is generally the case, the distribution of inclusions is isotropic as well as homogeneous, the relation (7.10) must be invariant under spatial rotations as well as translations. This means that the effective dielectric tensor must be of the form

$$
\epsilon^{*}(\mathbf{q})=\epsilon_{l}^{*}(q) \hat{q} \hat{q}+\epsilon_{t}^{*}(q)(1-\hat{q} \hat{q})
$$

where $\epsilon_{l}^{*}$ and $\epsilon_{t}^{*}$ are the longitudinal and transverse dielectric constants, respectively, and $\hat{q}=\mathbf{q} / q$. In our case of an electrostatic field for which

$$
\operatorname{curl}\langle\mathbf{E}\rangle=0, \quad \text { or } \hat{q} \times\left\langle\mathbf{E}_{\mathbf{q}}\right\rangle=0
$$

only the longitudinal dielectric constant remains in (7.10).

We come now to the question of when the q-dependence of the dielectric tensor is important. The answer depends upon the form of the distribution of inclusions and to some extent on the nature of the inclusions 
themselves. At low densities, if the correlation length of the impurity distribution is of the order of the radius $a$ of an impurity, low $q$ means $q a \ll 1$. For many applications this will be the case and we can then set $q=0$ in the above expressions. This corresponds to assuming the mean field $\langle\mathbf{E}\rangle$ is uniform and in that case

$$
\epsilon^{*}(0)=\epsilon^{* 1}
$$

since $\epsilon_{l}^{*}(0)=\epsilon_{t}^{*}(0) \equiv \epsilon^{*}$. Using (7.9) in (7.11) and setting $q=0$, we find our desired expansion for $\epsilon^{*}$ :

$$
\epsilon^{* 1}=\epsilon_{1} 1+4 \pi \sum_{s=1}^{\infty} \frac{1}{(s-1) !} \sum_{(B)} \int \cdots \int d \mathbf{R}_{2} \ldots d \mathbf{R}_{s} b(B)(0|\mathrm{C}(B)| 0)
$$

where $b(B)$ is given by (5.12) and $\mathrm{C}(B)$ by (5.13). In (7.15) there is no need to set $\mathbf{R}_{1}=0$, since the integrals are independent of $\mathbf{R}_{1}$.

We conclude by writing out more explicitly the first few terms in the expansion (7.15). We have already seen that the term $s=1$ is given by (3.22). Using this we write

$$
\begin{aligned}
\epsilon^{* 1}= & \left(\epsilon_{1}+4 \pi n \alpha\right) 1 \\
& +4 \pi \int d \mathbf{R}_{2} n(1,2)(0|\mathrm{M}(1,2)-\mathrm{M}(1) \cdot \mathrm{L}(2)| 0) \\
& +4 \pi \int d \mathbf{R}_{2}[n(1,2)-n(1) n(2)](0|\mathrm{M}(1) \cdot \mathrm{L}(2)| 0)+\cdots
\end{aligned}
$$

\section{RELATION WITH VIRIAL EXPANSIONS IN STATISTICAL MECHANICS}

The formalism we have introduced based on a continuum picture of the medium applies as well to the problem of the dielectric response of a nonideal gas of polarizable molecules. If we set the dielectric constant $\epsilon_{1}$ of the background medium equal to unity, then the inclusions may be considered as a gas of spherical molecules in a vacuum. Within the framework of classical equilibrium statistical mechanics, the probability distribution $W(1, \ldots, N)$ introduced in Section 2 is then given by

$$
W(1, \ldots, N)=\exp \left[-\frac{V(1, \ldots, N)}{k_{B} T}\right] / Q(V, T, N)
$$


where $V(1, \ldots, N)$ is the total intermolecular potential energy and

$$
Q(V, T, N)=\int \cdots \int d \mathbf{R}_{1} \ldots d \mathbf{R}_{N} \exp \left[-\frac{V(1, \ldots, N)}{k_{B} T}\right]
$$

is the configuration integral for the canonical ensemble. There are a number of restrictions associated with this identification. The first is that we consider only the linear response. For intense electric fields there will be nonlinearities which, in part, can be associated with field-induced changes in the distribution (8.1). The second restriction is the nonoverlap requirement (2.10). This implies a molecular model with a hard-core radius $a$, inside which the polarizability of the molecule is confined. More explicitly this means that when an isolated molecule is placed in an arbitrary applied electric field, the induced polarization must vanish outside of $a$. There can, of course, be additional (van der Waals) intermolecular forces outside $a$. A third restriction is that the molecular polarizability is intrinsic: it is not affected by the presence of other molecules.

Within the framework of equilibrium statistical mechanics expressions have been derived by a number of authors for the so-called dielectric virial coefficients, ${ }^{(32)}$ defined as the coefficients in the density expansion of the Clausius-Mossotti function $\left(\epsilon^{*}-1\right) /\left(\epsilon^{*}+2\right)$. In our formalism this expansion can be obtained from the expansion (3.12) of the average polarization. If we assume the sample is spherical in shape and the applied field $\mathbf{E}_{0}$ is uniform, then from elementary electrostatics we know that the field within the sample is uniform, given by

$$
\langle\mathbf{E}\rangle=\frac{3}{\epsilon^{*}+2} \mathbf{E}_{0} \quad \text { (spherical sample) }
$$

But the average polarization and the field within the sample are in general related by

$$
\langle\mathbf{P}\rangle=\frac{\epsilon^{*}-1}{4 \pi}\langle\mathbf{E}\rangle
$$

Using these two relations in (3.12) we find for a spherical sample

$$
\begin{aligned}
\frac{\epsilon^{*}-1}{\epsilon^{*}+2} \mathbf{E}_{0}= & \frac{4 \pi}{3} \sum_{s=1}^{\infty} \frac{1}{(s-1) !} \\
& \times \int \cdots \int d \mathbf{R}_{1} \ldots d \mathbf{R}_{s} n(1, \ldots, s) \mathrm{M}(1 ; 2, \ldots, s) \cdot \mathbf{E}_{0}
\end{aligned}
$$

Since the distribution of molecules within the sample is homogeneous we may, as in Section 7, replace the $\mathbf{R}_{1}$ integration by an integration over the 
field point at fixed $\mathbf{R}_{1}$. Then, using the notation of Section 7 , we may effectively cancel $\mathbf{E}_{0}$ and write (8.5) in the alternate form:

$$
\begin{aligned}
\frac{\epsilon^{*}-1}{\epsilon^{*}+2} 1= & \frac{4 \pi}{3} \sum_{s=1}^{\infty} \frac{1}{(s-1) !} \int \cdots \int d \mathbf{R}_{2} \ldots d \mathbf{R}_{s} n(1, \ldots, s) \\
& \times(0|\mathrm{M}(1 ; 2, \ldots, s)| 0)
\end{aligned}
$$

The coefficients in this expansion are strictly speaking not the dielectric virial coefficients. These would be obtained by expanding the partial distribution functions in powers of the density, using the well-known virial expansions for the nonideal gas, and rearranging ${ }^{(27)}$ according to powers of $n$.

The shape dependence of the result (8.6) is seen in a dramatic way if one applies the arguments of the preceding paragraph to a sample in the form of a plane slab. The result is an expansion of the function $\left(\epsilon^{*}-1\right)$ $13 \epsilon^{*}$, different from the Clausius-Mossotti function, with however a righthand side which is formally identical with the $z z$ component of the right-hand side of (8.6), if the slab is perpendicular to the $z$ direction. The two expansions must give the same result for $\epsilon^{*}$ when carried out for the appropriate sample shape, since our expansion (7.15) ensures the existence of a shape-independent $\epsilon^{*}$.

The earlier formulations of Buckingham and Pople ${ }^{(10)}$ and Mandel and Mazur ${ }^{(33)}$ are equivalent to the expansion (8.6) with the canonical distribution (8.1). Later formulations by Hill, ${ }^{(11)}$ Kaufman and Watson, ${ }^{(12)}$ and Isihara ${ }^{(13,14)}$ lead to the same expansion but employ the grand canonical ensemble either in classical or quantum statistical mechanics to replace (8.1). In these calculations the molecules are generally treated as being electrically pointlike, having only a dipole polarizability, although refinements including quadrupole corrections have appeared. ${ }^{(34-36)}$

\section{DISCUSSION}

We have derived a cluster expansion for the electrical susceptibility kernel of a dielectric suspension and have shown that it can be written as a sum of cluster integrals, each of which is absolutely convergent. As a consequence, in the bulk of a large sample there is a well-defined dielectric constant which is independent of the shape of the sample. We have shown the relation to previous virial expansions in statistical mechanics, which involved cluster integrals which are only conditionally convergent.

In following papers we shall further analyze the cluster expansion. In particular we shall show that our second-order integral is equivalent to Finkel'berg's ${ }^{(20)}$ and Jeffrey's ${ }^{(21)}$ and we shall also make a comparison with 
Jeffrey's higher-order terms. ${ }^{(23)}$ Furthermore it will be shown how to make a selection of terms which leads to the Clausius-Mossotti formula and how to obtain corrections to that formula.

In conclusion we note that our formalism is quite general and can be applied with minor modifications to multicomponent systems and to nonspherical particles. Also many other transport problems in disordered systems can be treated with the same method.

\section{ACKNOWLEDGMENT}

We thank the NATO Research Grants Programme for grant No. 1945 to support this research.

\section{REFERENCES}

1. G. K. Batchelor, Ann Rev. Fluid Mech. 6:227 (1974).

2. R. Landauer, in Electrical Transport and Optical Properties of Inhomogeneous Media, AIP Conf. Proc. 40, J. C. Garland and D. B. Tanner, eds. (AIP, New York, 1977), p. 2.

3. R. Herczynski and I. Pienkowska, Ann. Rev. Fluid Mech. 12:237 (1980).

4. E. Kröner and K.-H. Anthony, eds., Proceedings Third Int. Symp. on Continuum Models of Discrete Systems, Freudenstadt, June 1979. (University of Waterloo Press, Waterloo, Canada, 1980.)

5. W. F. Brown, Jr., Handbuch der Physik, S. Flugge, ed. (Springer, Berlin, 1956), Vol. XVII, p. 1.

6. J. G. Kirkwood, J. Chem. Phys. 4:592 (1936).

7. J. Yvon, Recherches sur la théorie cinétique des liquides: I, Fluctuations en densité (Actualités Scientifiques et Industrielles No. 542) (Hermann, Paris, 1937).

8. D. Bedeaux and P. Mazur, Physica 67:23 (1973).

9. B. U. Felderhof, Physica 76:486 (1974).

10. A. D. Buckingham and J. A. Pople, Trans. Faraday Soc. 51:1029 (1955).

11. T. L. Hill, J. Chem. Phys. 28:61 (1958).

12. A. N. Kaufman and K. M. Watson, Phys. Fluids 4:931 (1961); J. Chem. Phys. 36:439 (1962).

13. A. Isihara and R. V. Hanks, J. Chem. Phys. 36:433 (1962).

14. A. Isihara, J. Chem. Phys. 38:2437 (1963).

15. M. S. Wertheim, Mol. Phys. 25:211 (1973).

16. J. D. Ramshaw, Physica 62:1 (1972).

17. G. W. Ford in Ref. 4.

18. E. G. D. Cohen, "The Kinetic Theory of Dense Gases," in Fundamental Problems in Statistical Mechanics II, E. G. D. Cohen, ed. (North-Holland Publishing Co., Amsterdam, 1968), p. 228.

19. V. M. Finkel'berg, Sov. Phys. JETP 19:494 (1964).

20. V. M. Finkel'berg, Sov. Phys. Dokl. 8:907 (1964).

21. D. J. Jeffrey, Proc. R. Soc. London Ser. A 335:355 (1973).

22. G. K. Batchelor, J. Fluid Mech. 52:245 (1972).

23. D. J. Jeffrey, Proc. R. Soc. London Ser. A 338:503 (1974). 
24. R. Kubo, J. Phys. Soc. Jpn. 17:1100 (1962).

25. N. G. van Kampen, Physica 74:239 (1974).

26. J. A. Stratton, Electromagnetic Theory (McGraw Hill, New York, 1941) p. $211 \mathrm{ff}$.

27. G. E. Uhlenbeck and G. W. Ford, "Theory of Linear Graphs," in Studies in Statistical Mechanics, Vol. 1, J. de Boer and G. E. Uhlenbeck, eds. (North-Holland Publishing Co., Amsterdam, 1962).

28. T. D. Lee and C. N. Yang, Phys. Rev. 113:1165 (1959).

29. E. H. Hauge and E. G. D. Cohen, J. Math. Phys. 10:397 (1969).

30. K. M. Watson, Phys. Rev. 105:1388 (1957).

31. R. J. Elliott, J. A. Krumhansl, and P. L. Leath, Rev. Mod. Phys. 46:465 (1974).

32. C. J. F. Böttcher, Theory of Electric Polarization, Vol. I, 2nd ed. (Elsevier, Amsterdam, 1973), Chap. 6.

33. M. Mandel and P. Mazur, Physica 24:116 (1958).

34. R. W. Zwanzig, J. Chem. Phys, 25:21 (1956).

35. A. D. Buckingham and J. A. Pople, J. Chem. Phys. 27:820 (1957).

36. D. A. McQuarrie and H. B. Levine, Physica 31:749 (1965); J. Chem. Phys. 44:3500 (1966). 